\title{
Study on the Tone and Aesthetics of Architecture
}

\author{
Yifan Guo ${ }^{1, *}$
}

\author{
${ }^{1}$ Wuhan Textile University, Wuhan, Hubei, China \\ *Corresponding author. Email:85464510@qq.com
}

\begin{abstract}
This article expounds from the multiple analysis of the architecture's virtual reality, tension, rhythm, confrontation, void, symbol, light and field. An extremely profound example analysis is used to explore the imagination of architectural aesthetics, and then it is further sublimated to the so-called architectural tone on the spiritual level, providing some methods and guidance for people who love and appreciate the beauty of architecture.
\end{abstract}

Keywords: mass, equilibrium, landscape construction, symbol, light

\section{INTRODUCTION}

The form of architecture is the architect's language. For architecture, form is its life, and life has characteristics, and the simplest form can often convey the most distinctive characteristics. As long as matter is constructed in space, mass is produced, so the form of mass of matter and the adjacent space form a fitting relationship, which is being real and being virtual. If people want to make the building come alive, the first step to consider may be to get out of equilibrium and find a rhythmic order. Tradition is by no means just form, and harmony should not be repetitive. Only with a sense of confrontation can colorful forms be produced.

\section{MinimalisM}

The shape of the building is the language of the architect. This is the same as painters and sculptors. With the same intellects, they may have different temperament. And there is also a distinction between conciseness and prolixity. For example, the shape of Beijing West Railway Station is very stupid, large and improper; and the shape of the Military Commission Building is very appropriate, simple and magnificent. They are all made by Chinese people, and they are different in the shape. The design for the roof of the skyscraper makes the architects have a headache. Among many design for the roofs such as the squareshaped roof, round roof, single-tower roof, doubletower roof, it's still difficult to see a design needed. leoh Ming Pei made the Bank of China Tower, beautiful and magnificent. Even in Hong Kong, the Bank of China Tower is still simple and distinguished among the high-

*Fund: Youth Project of Philosophy and Social Science Research of Hubei Provincial Department of Education - Research on the Innovative Application of Chu Culture Elements in the Design of Modern Space Environment (No.: 19Q088); Teaching Research Project of Wuhan Textile University - Research and Practice of Modeling Design in Art Design Specialty (No.: 2018JY070) rise buildings. Mr. Pei's thinking about architecture is worthy of serious study. It seems that he has been speaking in simple language. His works are not in the style of "less representing more". However, these works are in the form of absolute minimalism. Whether these works are closed or open, they all reflect the minimalism. As for the glass pyramid in front of the Louvre Museum, it looks like nothing at the first glance, and the invisible is better than visible with detailed thinking. The use of a transparent "hidden" word solves the functional problem and avoids disputes such as style and context. This is great wisdom. Mr. Pei is a master in term of using rulers. His formal language is straight, without any mess. There are also people who do not want to use rulers to make the Sydney Opera House, Yoyogi Stadium, and the Millennium Dome, all of which leave a glorious mark on the history of architecture. The shapes of many good buildings in modern times can be drawn with a simple stroke. The "minimalism" should be an architects' fantasy about the architecture in the manuscript stage. Many people will not make manuscripts. If the architects are not good at finding suitable options from thousands of manuscripts, it will prove that the research on form is not enough. When the architects make a lot of manuscripts, he will find that the simplest form is the best, and the simple form can evoke a power when looking back. The beginning of architecture is the shape. For architecture, shape is its life. Life has characteristics, and the simplest form can often convey the most distinctive characteristics. Then, having the manuscript should be the first step for the architect to design the new building.

\section{TENSION}

As long as matter is constructed in space, physical volume is produced. The volume form of matter and the adjacent space form a fitting relationship, which is real and virtual. In human vision, the real part expands to 
the imaginary part, which means that the body surface of the solid part protrudes toward the adjacent void. The expansion movement that can be felt by this vision is the spatial tension of the physical form. Sculpture takes the tension as a basic modeling element. It can be said that sculptors who do not understand the management of salient point are not sculptors. Most architects are familiar with this. All are committed to the form tension of architecture. Eiffel Tower, CASA MILà, Mendelssohn Observator, Wright's Flowing Water, Ronchamp Church, Parliament House of Niemeyer, Nakagin Capsule Tower of kisho kurokawa, Sydney Opera House of Utzon, Sri Lanka Tallinn Library, De Fons Arches of Spreckson, Guggenheim Museum of Gehry ... are buildings with formal tension. The convexity of the building creates the concave. Under the action of light, the concave part is the shadow. As long as the building manages the shadow, the building form has a thickness in addition to the tension. Tian'anmen Square has a strong upward form tension, but Great Hall of the People doesn't have the tension. Except for the flat top, the vertical depth and projection are the main reasons. Shanghai Grand Theater and Shanghai Museum are located in the same square. The Grand Theater is clear and there is a strong tension, but the Museum has no tension. It is like a sunny young girl standing with a tightly wrapped old man. Which is dazzling?

Sculptors are experts in creating concavity and tension. Architects may wish to learn from sculptors with an open mind.

\section{BEING VIRTUAL}

When people look at architecture, they see the visible volume as the real and the invisible or unclear volume as virtual. The reason for this feeling is the projection of light and black holes. With the virtual, the building has a sculptural volume and thickness. General architects do not deliberately seek this sculptural language. They often inadvertently form this convexconcave relationship on the surface of the building according to their functional needs. Due to the effect of light, these convex-concave forms a real-virtual impression visually.

If the architects deliberately pursue this real-virtual relationship in the design, a new scene may be created.

Corbusier's chapelle de ronchamp deliberately operated the light projection and the dense arrangement of the windows, which showed his skill in mastering the shape of the building. The windows of different sizes seemed to be scattered on the concrete wall randomly. This anti-conventional window-opening method of the building made the facade of chapelle de ronchamp extremely vivid and spiritual. The thick large wall isolates the society, and the atrium is dark. Through the windows on the outer wall, the sun casts a cascading light waterfall on the church. The believers are sanctified by the wonder of the light. Natural light is here the light of God.

The chapelle de ronchamp is a classic that wins from the virtual. It is necessary to learn from it.

If the architects want to suspend large buildings, the "virtual" treatment of the lower part may be a solution.

If the architects want to make some walls of the building into floating white clouds, doing the "virtual" treatment may be a way.

If the architects don't want to make the corner walls to be bumped against each other, doing the virtual may be an excellent idea, and the result may be the opposite of the way of deconstruction. The thick wall seems to protrude from the inside, conveying the great power of the structure.

There are many results.

Only in this way can many new faces appear in the building.

\section{RHYTHM}

Equilibrium is the most general order of the building. Balanced floors, balanced windows, balanced columns ... are true from the flat to the vertical. The balance is like a $2 / 4$ beat, and the rhythm is flat and simple.

Uneven combination arrangement is rich in movement rhythm. For example, when Venus lifted his hip, one side of his body was squeezed, and the other side stretched. The posture of the human body reflects the rhythm of movement in this relaxation. In the calligraphy composition, "horse racing in the space matching with the density" refers to a rhythm of positive and negative arrangement in a flat space. Rich in the movement rhythm, it can often evoke the association of poetry and passion. When people enter this emotional feeling, they are hyperactive or excited.

If the architects want to make the building alive, the first step to consider may be to go out of equilibrium and find a rhythm of movement. This rhythm comes from the combination of different spatial forms and the control of building components in the arrangement. This ability refers to the architects' understanding and expressiveness of the rhythm (such as denseness, stretch, size, height, reality and transparency....)

A building has different spatial units. For example, a museum has several functional systems for storage, display, restoration research, and operation management. These functional systems have different space requirements. There are two ways to build a museum. One is to determine a uniform column network and floor height, fences, windows, and roofs. 
Marking the functional scope on the plane is like making a workshop. What general architects can embody is the characteristics of the walls and roofs. These are various museums what people see, but they are similar. This is the $2 / 4$ beat, lacking poetry and passion.

Second, according to different functions, different space units are constructed, and then these different space units are combined. Different functional spaces are constructed in different ways. For example, ceramic treasures and dinosaur skeletons are on the same streamline, and they are also exhibition halls. Their construction methods should be different. The space forms of storage, exhibition halls, and office buildings are also different. Different spatial forms provide different scales and shapes. This organic combination of different scales and forms will produce the rhythm of the building. The walls and windows are densely arranged, and the raised and recessed parts of the building are virtual and real, and the rhythm of the building appears naturally.

Regarding the rhythm of the building, the architect should listen more to symphonies. Whether classical or modern, museum buildings are a symphonic poem. What about a villa? What about a skyscraper? Symphonic poems are also symphonic poems in the author's understanding. Music, poetry and architecture are all connected in the proposition of rhythm.

\section{CONFrontation}

Confrontation refers to the relationship between a building (and landscape construction) and the original conditions (referring to an existing natural condition or the surrounding environment). The word corresponding to confrontation is harmony. Emphasizing harmony will make the building engulfed by the existing formal environment, lack of personality. Confrontation is just the opposite. It is a very different existing way corresponding to the existing environment. For designers, confrontation represents the design consciousness, which stimulates a personalized creative labor.

The buildings of leoh Ming Pei are almost buildings with the confrontation design consciousness. He has added many era marks to this world. Paris has unparalleled European classic architecture, but Paris does not seek the harmony between new architecture and classics. Instead, it has the Pompidou Center, De Fence Gate, La Villette Park and Music City. These new buildings, which are very different from the classical style, that prove to the world that Paris is on the move. This is the success of confrontation consciousness. What about Beijing? The foreigners say that there is no (new) building. That's so outrageous. However, as long as people look at the large and small pavilions and antique decoration in the city, it has to submit to the harsh comments of the Westerners. Many clumsy nondescripts are the result of harmony.

Today's Paris has sealed the past, but inherited the romance and exquisite feelings of the French.

Beijing has picked up the bits and pieces of the years, but has lost the magnanimity and creativity.

Tradition is more than just form, and harmony should not be repetitive.

Only with the consciousness of confrontation can colorful forms be produced, and confrontation does not prevent spiritual tradition from continuing.

\section{VOID}

Moore is a great contemporary sculptor. He is good at cutting various holes in the sculptures, such as the famous "Emperor and Queen". Moore's greatness lies in the fact that he penetrated the core of the sculpture and used the void to shape the core. Before this, sculptures were always clumped, and the objects they shaped were shapes. There was no use of space to shape the inner part of life forms. The building has always been a clumsy landscape for the city, but Spriksen and Andrew of Denmark made a huge arch for Paris. And a $60 \mathrm{~m} \times$ $80 \mathrm{~m}$ cavity was made in the center of the building. A huge pull-membrane net is suspended below the void, like a flowing cloud in the blue sky and a holy island in the sea. This government office building is no longer a majestic mass; it has been poeticized, and has become a world-renowned urban landscape. This should be a stunning romance created by an unparalleled imagination.

The building in China is not poetic or romantic, and even learning to make the void is not poetic or romantic. People look at architecture and cannot imagine anything else. Is the house too solid for Chinese nation stressing the concept of "empty"? Is the land too full? It's congested when everything is done. Therefore, cavity is a topic that requires urgent research for architects and planners.

\section{SYMBOL}

When human beings did not have steel and cement, the solution of building spans can only be achieved by the technology of wooden frames and buckets, or stone arch technology. With the progress of civilization, various building components made with these technologies have been decorated or simply evolved into pure architectural decoration, such as columns, corridors, doors, windows, canopies, double eaves, bucket arches, slope crest, spires , arch coupons, pediment and so on. The use of these component decorations represents the status of different classes in different regions and at different times. Later generations regarded these things as cultural symbols. 
When human beings already have the construction technology to solve the span and height, there are more and more large and tall houses. People finally look tired of these glittering cold square boxes. Some architects set their sights on these symbols of the splendid old architecture, borrowed these symbols to decorate the wall, and opened up the distance from modern architecture. Critics called it post-modern architecture, with the emotional comments such as the "context" and "respect for tradition".

However, the wall is decorated, and the inner capsule is still the space structure of modern architecture. The so-called post-modern is only a piece of skin, and a flat decoration. The "skin" is not the "context". It's a big deal.

Of course, post-modern architecture also has good works. Johnson's AT \& T building shows that he has a serious attitude to classical architectural forms and a very strict approach. With the East learning from the West, postmodernism is precious for China. For example, the Bank of China in Hankou directly copied the Bank of Frankfurt, and a podium with Roman columns was added. Stained glass of the tall buildings is cut wildly. The houses in Beijing are even more terrific, and all kinds of non-descript pavilions are on the roof. As long as you walk across the land of China, you pass the construction site fence surrounded by developers; it says "garden of European style". It is not necessary to discuss what is" European". This is just a pure commercial sensation. The decorative elements of exterior wall used are some simple classical architectural symbols, such as pediment, arch coupons, Roman columns, iron window bars and Venus and her sisters in white cement. "Europe" settled here. In the past few years, the author drove long distances and saw that the roadside shops were all horrible brands such as "Night Shanghai Hotel" and "Dream Paris". In fact, there were just two or three tables and floor tiles. However, this was the host's imagination of Shanghai, Dream Paris and Grand Hotel. Is the prevalence of postmodern symbolism in large, medium and small cities the imagination of European style by businessmen, owners and leaders or kitsch of design professionals? When the people that buy a house took a fancy to "continental" style, discover it is not "Europe style" and the use space that does not have much to win those careful calculation residential projects, they will realize that they spent a lot of money on wall symbols.

Symbols are nouns made by people who have cultural literacy. These symbols can be used in the cultural circle, but once they are sprinkled into the public, they are like a mist of water. The doctrine could have been created. Moreover, the entanglement is confusing. Therefore, the architect must not start from the doctrine to make a house, otherwise the person who proposes the doctrine can do it.
Culture can be inherited. Some of the spirits generated by architecture can also be passed on, but that was after the formation of the architecture. The architecture has nothing to do with culture. What architecture needs is science. Therefore, the influence of cultural definitions (including symbols) can be eliminated to the fullest extent, and the scientific spirit of architecture can be carried out completely based on the interests of the people. This is technology, system, rationality, and refinement (sometimes rough and delicate).

\section{MaLALIGNMENT}

The basic rules of house structure are parallel. The column is parallel to the column, the level is parallel to the level ... the house is drawn with a parallel ruler plus a vertical triangle. The vast majority of houses around the world are built according to this rule. However, some people did not follow this rule and made some non-parallel houses. Such as the Dulles Airport Terminal in Shalinin, Kenzo's Gymnasium, Utzon's Sydney Opera House, Sterling's Art Gallery, Guggenheim Museum of Wright and Gehry come to life, just like the natural rocks on the Holy Land, or the fluttering birds, or the sails, or nothing at all, unbelievable but strange and even dreamlike. The extraordinary imagination and courage of the architects left a string of pearls for the 20th-century architecture, and their original intention was to escape the parallel rules. These non-parallel buildings should be successful examples of transcending the rules, which open up new design ideas for public buildings.

\section{LIGHT}

With light, there is color. With light, bumps and shadows of volume are created, so sculpture becomes the art of volume and light. With the light, the golden magnificence of the Acropolis reflected in the setting sun. It is also because of the light that Corbusier's rough concrete wall reflects the epic symphony. Almost all masters of architecture are experts in using light. In their eyes, all materials are not inherent colors. Using the effect of light on materials to create tranquility or purity, producing dignity or magic, is their specialty.

Therefore, architecture began to move from building to "playing" buildings, just like the artist "playing" with colors and volumes. Of course, this is not cynical, but the natural manifestation of the masters' extraordinary abilities.

Nowadays people attach great importance to valuing natural resources, such as landscapes, forests, ozone layers ... What about sunlight? Apart from solar experts, few people think of light as a natural resource. People have neglected the generosity of light to human beings. This is also the case for many architects. The depth of 
the house is getting bigger and bigger. It is very easy to go up and down, but without light, it can only rely on artificial lighting. Staying in such a house for a long time, people will feel very tired. The eyes can't stand because of the forced lighting for a long time. When people walk out of the office building and embrace the setting sun after a day of work, it is like the end of a sleepless night. The office building is relying on artificial lighting, and day likes night. A person "works night shift" for months and months. Smart buildings are our big goal now, so what about sunny buildings? If people choose between plot ratio and health, it believes that health should be preferred. The architectural philosophy has evolved from the settlement project to the pursuit of space quality, and architecture should have been people-oriented. Ignoring people-oriented architecture is a short-sighted economic act. Getting close to the sun is a new architectural topic and a new selling point for commercial buildings.

\section{FIELD}

After the building is completed, its form and volume can make people get a certain spiritual feeling within a certain spatial range. For example, the military commission building on the east side of the Beijing Military Expo is magnificent, showing a proposition of architecture like a boulder standing still. Another example is the Sun Yat-sen Mausoleum in Nanjing, where people can feel the forerunner's longevity without having to visit it. Like the Golden Dome of Wudang Mountain, as long as people go around, people will appreciate what is good. Forbidden City, Suzhou Gardens, Zhouzhuang, Yixian ... can all give people a variety of feelings, such as the king's style, delicate feelings, indifferent leisure or plain elegance ... Architecture controls the space and gives people a variety of spiritual feelings. This kind of space is the field of architecture, and the material has completed the transformation into spirit in the field.

Not all spaces have this kind of field. If people put the golden dome in the Imperial Garden of the Forbidden City or the Liudu Bridge in Hankou, it will not be possible to get a spiritual shock. The space that can be called a field is the result of the mixed action of architecture and the environment. This environmental condition is not arbitrary and can be replaced and imitated.

Not every building has the ability to form a space field. Generally, the larger the building mass, the more likely it is to form such a field. But there are also small and ingenious examples. For example, on the road from Nanyue Great Temple to Daxiong Baodian, there is a very low-covered stone alley with unique ingenuity. Here the civilian officer disembarks, the military officer dismounts, and even people who are less than $1.6 \mathrm{~m}$ tall shrink the heads. When people have finished walking this stone alley, all the hustle, irritability, lightness, and leisure are gone, and everyone is calmly walking towards the temple of the Buddha. It is this extremely low-covered stone alley that transforms the earth into the upper bound. There is also the "Rebirth Hall" under the side of Wudang Jinding, which is actually a concave lane with a width of about $40 \mathrm{~cm}$ and a length of about $10 \mathrm{~m}$. When people enter the lane, there is no light. People can move sideways only by closing the chest and back. At this time, an old man kept banging on the gong and asked, "Have you come here?" You must keep answering "here". People are scared in the dark and narrow space, and the voice of "coming over or not" in the midst is like a fairy calling. When people suddenly see the sun again, they will have life again. Of course, people are willing to present a banknote to the merit box

\section{CONCLUSION}

The building can express emotions and make the space in which it is located magical. This requires that the person who constructs the building must know how to use psychoanalysis to let his work talk to the public, and then his work will produce a field.

Having talked a lot, the author doesn't talk about doctrine or criticism. Most of the judgments during this period are based personal preferences and ridicule. This is what the so-called tone. It is necessary to keep and stick to the aesthetics.

\section{References}

[1] (Denmark) Jan Gehl, Life Between Buildings [M]. He Renke, trans. Beijing: China Architecture \& Building Press, 2002. (in Chinese)

[2] (Italy) Umberto Eco, Storia Della Bellezza [M]. Peng Zedong, trans. Central Compilation \& Translation Press, 2007. (in Chinese)

[3] Philip Jodidio, Janet Adams Strong, I. M. Pei Complete Works [M]. Li Jiajie, Zheng Xiaodong, trans. Publishing House of Electronics Industry, 2011. (in Chinese)

[4] Le Corbusier, LE POÈME DE L'ANGLE DROIT [M], Sichuan Literature and Art Publishing House, 2019, (04): 5-7.

[5] Xu Ning, I. M. PEI And Suzhou Museum [M]. Suzhou Gu Wu Xuan, 2007, (9): 4-5.

[6] Tadao Ando, Tadao Ando: to Build a World of One's Own [M], CITIC Press Group, 2012, (2): 1-2.

[7] Wang Shaoqiang, Designer's Code 100 [M]. Guangxi Arts Publishing House Co. Ltd., 2017, (21): 1-2

[8] Carsten Krohn. Mies Van Der Rohe - The Built [M]. China Architecture and Building Press, 2018, (4): 28.

[9] Thom Mayne. Combinatory Urbanism [M]. Phoenix Science Press, 2019, (4): 49 